\title{
Inaugural ACNS Highlights Recent Developments in Interdisciplinary Science using Neutron Scattering
}

The inaugural American Conference on Neutron Scattering (ACNS) was held June 23-27 in Knoxville, Tenn. The conference was organized by the Neutron Scattering Society of America (NSSA) and chaired by Rob Briber of the University of Maryland, Julie Borchers of the National Institute of Standards and Technology (NIST), and Paul Butler of Oak Ridge National Laboratory (ORNL). Attended by over 400 scientists and engineers from 13 countries, the multidisciplinary ACNS was sponsored by the national neutron centers with assistance from the National Science Foundation and the U.S. Department of Energy (DOE). The conference included 116 talks and 138 posters presented in the fields of biology, soft condensed matter, magnetism, instrumentation, fundamental neutron physics, chemistry, and industrial applications, all with the common thread of using neutrons for research. The ACNS also served as a national meeting for present and potential users of the neutron research centers at Argonne National Laboratory, Los Alamos National Laboratory (LANL), NIST, ORNL, and Chalk River (Ontario, Canada), and provided an opportunity to gain insight into the capabilities and available instrumentation at these facilities. Tours of the Spallation Neutron Source Project and the High Flux Isotope Reactor at ORNL were also available. The NSSA plans to hold the ACNS every two years as a showcase for developments in science using neutron scattering.

The plenary talk of the conference was given by Robert Cava of Princeton University on current directions in materials research, in which he outlined many new systems where neutron scattering is having a significant impact. Additional presentations included an invited talk by Norm Wagner of the University of Delaware on combining small-angle neutron scattering (SANS) and rheology for understanding the structure of colloidal systems. Jill Trewhella of LANL discussed the role of neutron scattering in elucidating the structure of complex biological assemblies composed of multiple proteins. The application of neutron scattering with deuterium contrast labeling is a powerful technique in combination with synchrotron $x$-ray scattering for understanding these biological assemblies. Hubert E. King of Exxon discussed the impact of neutron scattering on

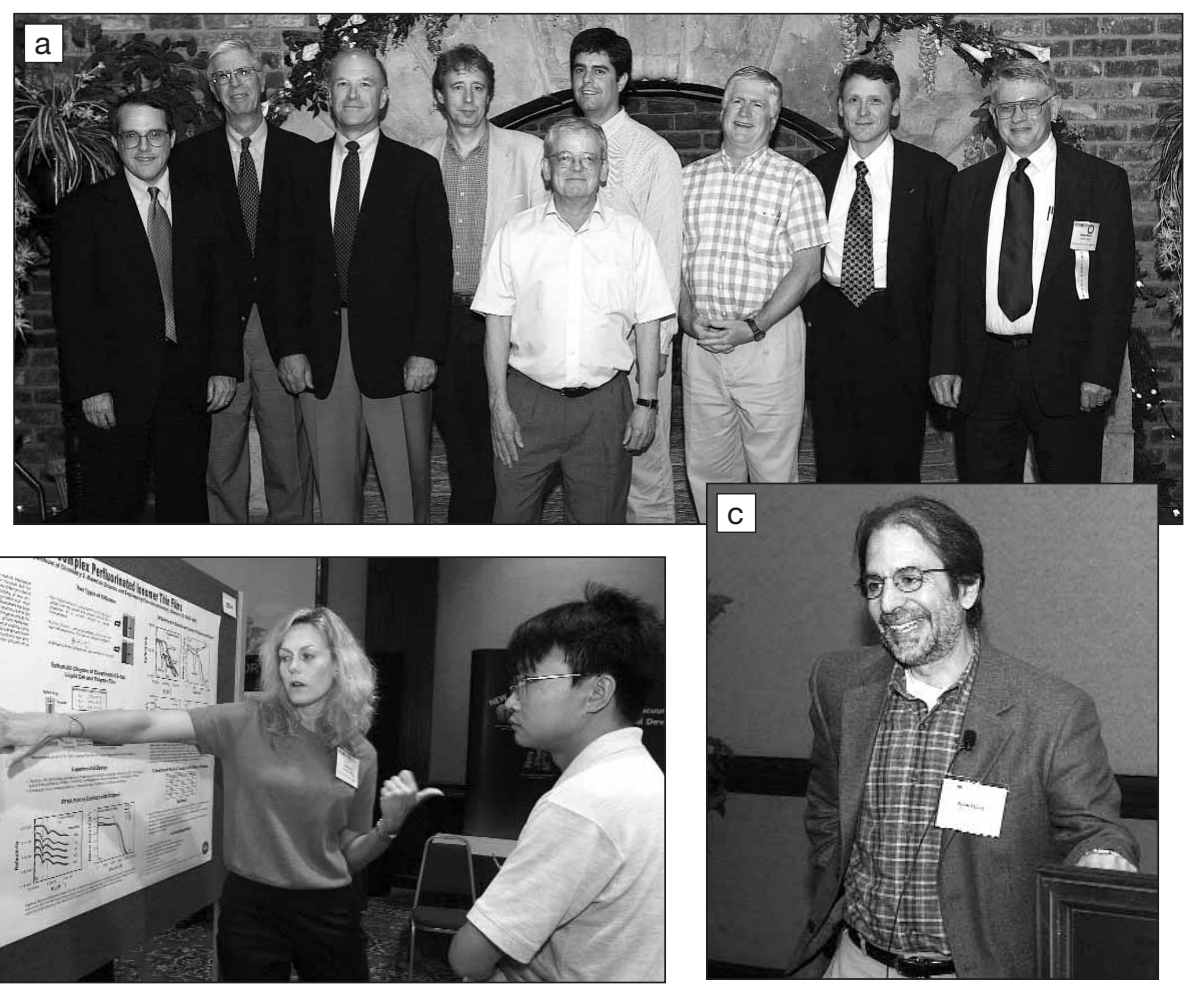

(a) Directors of the U.S. neutron scattering centers, chair of the American Conference on Neutron Scattering, and president of the Neutron Scattering Society of America: (left to right) Jim Roberto (Oak Ridge National Laboratory), Herb Mook (Oak Ridge National Laboratory), Paul Lisowski (Los Alamos Neutron Scattering Center), Rob Briber (University of Maryland), Michael Rowe (National Institute of Standards and Technology), Thom Mason (Spallation Neutron Source), Ray Teller (Intense Pulsed Neutron Source), Alan Hurd (Los Alamos Neutron Scattering Center), and James Rhyne (University of Missouri). (b) Teresa A. Hill (Clemson University) discusses her poster with Zhibin Li (University of Tennessee) on solvent diffusion into ionomer thin films. (c) Robert Cava of Princeton University gives the plenary talk at the inaugural American Conference on Neutron Scattering.

the oil industry and its specific role in oil production. Barbara E. Wyslouzil of Worcester Polytechnic Institute described a challenging SANS measurement of nanodroplet aerosols formed in supersonic expansions. Fred E. Wietfeldt of Tulane University provided an overview of neutron physics with a specific emphasis on neutron $\beta$ decay. Nanostructured materials are a topic of broad current interest, and Ivan K. Schuller of the University of California, San Diego, focused his presentation on the contributions made by neutron scattering in the area of nanomagnetism. In particular, he described key insights that have been gained on interlayer coupling in magnetic multilayers, reversal mechanisms in exchange-biased systems, and the separation of magnetic and structural interfacial roughness. Another area of great interest at the conference was in new instrumentation and techniques being developed in anticipation of the Spallation Neutron Source currently under construction at ORNL. Ferenc Mezei of LANL and the Hahn-Meitner Institute presented an entertaining and thought-provoking talk titled "Free-Lunch Theorem in Pulsed Source Instrument Optimization." His ideas involved the manipulation of phase space to increase data rates for time-offlight neutron scattering measurements.

In addition to the scientific highlights of the conference, NSSA announced the establishment of the Clifford G. Shull Prize in Neutron Science of $\$ 5,000$ to be awarded every two years at future ACNS conferences. Shull shared the 1994 Nobel Prize for neutron scattering with Bertram N. Brockhouse. ACNS Co-Chair 